\title{
Spinal Trauma and Spinal Cord Injury (SCI)
}

\author{
Luc van Den Hauwe, Pia C. Sundgren, \\ and Adam E. Flanders
}

\section{Learning Objectives}

- To understand the prevalence and clinical consequences of spinal trauma.

- To appreciate the utility of radiography, computed tomography, and magnetic resonance imaging in the evaluation of spinal trauma and spinal cord injury.

- To comprehend the grading systems used in spinal trauma.

- To appreciate the soft tissue components of spinal trauma and how they differ in the pediatric population.

- To understand the imaging features of spinal cord injury and traumatic vascular injury.

\subsection{Imaging Modalities for Spinal Trauma}

In the emergency setting the appropriate selection of imaging for spinal trauma depends upon several factors such as, modality availability, the patient's clinical and neurological status, type of trauma (blunt, single, or multi-trauma), and other associated co-morbidities. Clinical factors to consider also include the quality and severity of pain, limitations in motion, or the presence of permanent or transient neurological deficits. MRI is reserved for those patients with post-traumatic myelopathy (spi-

L. van Den Hauwe

Department of Radiology, Antwerp University Hospital,

Antwerp, Belgium

\section{P. C. Sundgren}

Institution for Clinical Sciences/Radiology, Lund University,

Lund, Sweden

e-mail: Pia.sundgren@med.lu.se

A. E. Flanders $(\triangle)$

Department of Radiology, Thomas Jefferson University Hospital,

Philadelphia, PA, USA

e-mail: adam.flanders@jefferson.edu nal cord dysfunction) or in the instance whereupon a patient's symptoms that cannot be explained by findings on radiographs or CT, or when a reliable neurologic exam cannot be obtained.

\subsubsection{Plain Film Radiography}

In the rare circumstance where MDCT is not available, the initial imaging modality is radiography. A minimum of a lateral and anteroposterior view must be obtained for the spinal axis with the addition of an open-mouth odontoid view for the cervical spine. Often additional views such as oblique views and/or the swimmer's view are performed in an attempt to clear the cervicothoracic junction. With the exception of pediatric trauma, in most settings, radiography has been supplanted by MDCT.

\subsubsection{Computed Tomography (CT)}

Thin section multi-detector computed tomography (MDCT) is the preferred method when evaluating the cervical spine for bony injury after blunt trauma. The entire spinal axis can be reliably and expeditiously evaluated with automatic reformatting of the axial dataset into multiple planes allows for better and more exact diagnosis of bone- and soft tissue abnormalities [7-13]. Moreover in the instance of polytrauma, spine images can be reconstructed directly from chest, abdomen, and pelvis datasets with sensitivity that is equivalent to a dedicated spine CT study. This has the added benefit of minimizing radiation dose.

With the introduction of these new MDCT imaging techniques most trauma centers have set up dedicated acute (multi-) trauma protocol(s) which include CT of the brain, cervical spine, thorax, abdomen, and pelvis, with subsequent reformatting of images of the thoracic and lumbar spine. This both expedites the data acquisition for medically unstable patients and serves to minimize radiation dose since the body imaging data can be reconstructed offline into targeted spine reconstructions. 
CT has a higher sensitivity to fractures (especially involving the posterior elements) than radiography. This rapid cross-sectional imaging assessment of the spinal axis has been shown to be more efficient and safer by virtually eliminating the need for repeat radiographs and unnecessary patient transfers in the setting of an unstable spine. Moreover, the diagnostic quality of radiography varies considerably, is more time-consuming to acquire, and may be difficult to perform in a medically unstable patient. While MDCT excels at delineating bony injury, it also can detect many soft tissue abnormalities such as disc herniation, paravertebral soft tissue- and epidural hematoma. A highresolution CT imaging protocol begins with submillimeter overlapping partitions to create an isotropic dataset that yields identical spatial resolution in any reconstructed plane. Axial data can be reformatted into thicker sections for diagnostic display; with reformatted $1.25-2 \mathrm{~mm}$ thin slices in the $\mathrm{C} 1-\mathrm{C} 2$ region, 2-3 $\mathrm{mm}$ thin slices in the rest of the cervical spine, and 3-4 $\mathrm{mm}$ thin slices in the thoracic and lumbar spine are typically chosen for axial presentation. Reformatted sagittal and coronal images of the entire spine are produced from contiguous submillimeter $(0.3-0.75 \mathrm{~mm})$ axial images. Multiplanar reformatted (MPR) sagittal and coronal images of the entire spine are typically produced automatically from the scanning console or from a nearby workstation. Reconstructions are performed with both bone and soft tissue algorithms.

\subsubsection{Magnetic Resonance Imaging (MRI)}

The greatest impact that MRI has made in the evaluation of spinal trauma has been in assessment of the soft tissue component of injury. MRI is today considered the method of choice for assessing the spectrum of soft tissue injuries associated with spinal trauma. This includes damage to the intervertebral discs, ligaments, vascular structures, and spinal cord [14-16]. No other imaging modality has been able to faithfully reproduce the internal architecture of the spinal cord and it is this particular feature that is unique to MRI. Any patient who has a persistent neurologic deficit after spinal trauma should undergo an MRI in the acute period to exclude direct damage/compression to the spinal cord. MRI provides unequivocal evidence of not only spinal cord injury, but will also reliably demonstrate disc injuries/herniations, paraspinal soft tissue edema (ligament strain/failure), epidural hematomas, and vascular injury. In addition, MRI provides the most reliable assessment of chronic spinal cord injury and the imaging analogs of post-traumatic progressive myelopathy (PTPM) which is often manifested with imaging as syrinx formation, myelomalacia, and cord atrophy. The extent with which MRI is able to determine spinal instability is overstated as MRI is unable to provide a reliable assessment of ligamentous integrity in most cases. In fact, MRI falsely overestimates the soft tissue component of injury [17].

An acute spinal trauma MR imaging protocol of the cervical spine shall include $3 \mathrm{~mm}$ thick sagittal T1 (T1-weighted) and T2-weighted (T2W) and short tau inversion recovery (STIR) sequences and $3 \mathrm{~mm}$ thick axial T2*-weighted gradient echo (GRE) images without contrast. In the thoracic and lumbar spine, $4 \mathrm{~mm}$ thick sagittal T1-weighted, T2-weighted, and STIR sequences and axial $4 \mathrm{~mm}$ thick T1-weighted, T2-weighted, and T2*GRE images without contrast is recommended. 3D volumetric axial GRE or T2-weighted partitions at 1-2 $\mathrm{mm}$ thickness are useful in the cervical region. Fat-saturated T2-weighted images are valuable to evaluate for ligamentous and soft tissue injuries, and T2* GRE to evaluate for small hemorrhage or blood products in the spinal cord.

\section{Key Point}

- Radiography has largely been supplanted by MDCT except in the pediatric population for evaluation of bony injury.

\subsection{Different Grading Systems to Evaluate Spinal Injuries}

There are different classic grading scales for determining spinal instability of thoracolumbar injuries based upon the McAfee (two-column) and Denis three column concept [18, 19]. The Magerl classification relies exclusively on CT findings [20]. In recent years a new grading scale that is based on CT and magnetic resonance (MR) imaging findings, like the thoracolumbar injury classification and severity score (TLICS) has been developed by the Spine Trauma Group [21] to overcome some of the perceived difficulties regarding the use of other thoracolumbar spinal fracture classification systems for determining treatment. Also for the grading of the cervical spine a new grading scale and score system-the cervical spine Subaxial Injury Classification and Scoring (SLIC) system [22] - has been developed and is gaining acceptance among spine surgeons. The AO Spine classification system provides a comprehensive classification schema for upper cervical, subaxial cervical, thoracolumbar, and sacral injuries [23].

\subsubsection{Injuries to the Vertebral Column}

Classically, injuries to the spinal column are categorized by mechanism of injury and/or by instability. Instability is defined by White and Punjabi as abnormal translation between adjacent vertebral segments with normal physiologic motion. Unrecognized instability after trauma is a potential cause of delayed spinal cord injury. This is why early stabilization of the initial injury is an imperative to appropriate clinical management. The simplest method to test for instability in a controlled environment is by performing flexion and extension 
lateral radiography to produce a visible subluxation at a suspected level but this is rarely performed in practice.

From biomechanical point of view, the thoracolumbar spine can be divided into three osteo-ligamentous columns: anterior-, middle-, and posterior column [18]. The anterior column includes the anterior longitudinal ligament and anterior two-thirds of the vertebral body and disc including annulus fibrosus. The middle column is composed of the posterior third of the vertebral body and disc including annulus fibrosus, and posterior longitudinal ligament. Finally, the posterior column is composed of the pedicles, articular processes, facet capsules, laminae, ligamentum flavum, spinous processes, and the interspinous ligaments. The mechanism of injury will result in several different types of traumatic injuries to the cervical, thoracic, and lumbar vertebral column and spinal cord, which may result in stable or unstable spine injuries. Although this biomechanical model is often inferred for cervical injuries, there is no similar established model in the cervical spine.

Because of the distinct anatomic differences and the resultant injury patterns, injuries to the cervical spine are divided into subaxial injuries (cranial base to axis) and lower cervical injuries (C3-C7). The mechanism of injury to the cervical column can be divided into four major groups: hyperflexion, hyperextension, rotation, and vertical compression with frequent variations that include components of the major groups (e.g., flexion and rotation). Hyperflexion injuries include anterior subluxation, bilateral interfacetal dislocation, simple wedge fracture, fracture of the spinous process, teardrop fracture, and odontoid (dens) fracture. Of these the simple wedge fractures and isolated spinous process fractures are considered initially stable, while the other fractures are considered unstable such as the bilateral interfacetal dislocation and the teardrop fracture. The odontoid fracture can be considered stable or unstable depending on the type of fracture type. Hyperextension mechanism is less frequent than the hyperflexion and result in the following types of injuries: dislocation, avulsion fracture or fracture of the posterior arch of $\mathrm{C} 1$, teardrop fracture of $\mathrm{C} 2$, laminar fracture, and traumatic spondylolisthesis of C2 (Hangman's fracture). Most of these injuries with the exception of Hangman's fracture are defined as stable fractures; however, this does not imply that these injuries should go untreated. The hyperextension injuries are often associated with central cord syndrome especially in patients with pre-existing cervical spondylosis and usually produce diffuse pre-vertebral soft tissue swelling. Vertical compression results in the Jefferson fracture which involves atlas and is considered unstable or burst fractures. A common site for injuries is the craniocervical junction (CCJ) and the atlantoaxial joint, which is the most mobile portion of the spine as it predominantly relies on a complex ligamentous framework for stability. The imaging findings of important CCJ injuries, such as atlanto-occipital dissociation, occipital condyle fractures, atlas fractures with transverse ligament rupture, atlantoaxial distraction, and traumatic rotatory subluxation, are important to recognize in the acute setting as for the patient management.

Fractures in the lower thoracic and lumbar spine differ from those in the cervical spine. The thoracic and lumbar fractures are often complex and due to a combination of mechanisms. The thoracic cage confers substantial biomechanical protection to the thoracic spine. Therefore, statistically, most injuries occur at the most mobile portion or the thoracolumbar junction where the thoracic cage ends. When injuries occur in the upper or middle thoracic spine it is usually a result of major trauma, e.g., high velocity trauma such as motor vehicular accidents. The most common fracture, at the thoracolumbar junction, is the simple compression- or wedge fracture (50\% of all fractures) which is considered stable. The remaining types of fractures among those the so-called seat belt injury, which can be divided into three subtypes: type I (Chance fracture) involves the posterior bony elements, type II (Smith fracture) involves the posterior ligaments, and, in type III the annulus fibrosus is ruptured allowing for subluxation are considered unstable fractures [24]. With the advent of the three-point restraint system in motor vehicles, these severe hyperflexion-distraction injuries have become uncommon. The most common of all thoracolumbar fractures-the burst fractures account for $64-81 \%$ of all thoracolumbar fractures. The burst fracture, which can be divided into five subtypes, is associated with high incidence of injuries to the spinal cord, conus medullaris, cauda equina, and nerve roots [25]. It is important to remember that a burst fracture involving anterior and middle column can be misdiagnosed as mere compression fracture on plain films and, therefore may be misinterpreted as a simple compression or mild wedge fracture that involves only anterior column. CT has improved characterization of these injuries.

\section{Key Point}

- Different classification schemas are utilized to describe injury patterns based upon mechanism, anatomic and biomechanical differences in unique areas of the spine.

\subsection{Soft Tissue Injuries}

\subsubsection{Traumatic Disc Herniation and Ligamentous Injury}

Traumatic disc injuries are caused by distraction and shearing with failure of the intervertebral disc. A direct injury to the disc is more common than post-traumatic disc extrusion. 
Traumatic disc herniation should be considered when the disc exhibits high signal on T2-weighted images especially when traumatic vertebral body fractures and/or ligamentous injury is present at the same level [13]. Extruded disc material may extend into the epidural or pre-vertebral space. When there is a gap between parts of the vertebrae or by increased signal in the ligament or adjacent structures on fat suppressed T2-weighted or STIR images, a ligamentous injury is suspected. Up to $25 \%$ of all cervical injuries will demonstrate signal changes in the posterior ligamentous complex. This finding does not equate with instability.

Ligamentous injury without underlying fracture in the cervical spine is rare [26]. Disruption of the anterior longitudinal ligament is associated with hyperextension mechanisms with associated injury to the pre-vertebral muscles and intervertebral discs and can be identified as interruption of the normal linear band of hypointense signal of the ligament on T1-weighted images. Hyperflexion and distraction forces may cause disruption of the posterior ligament complex which is manifested by increased distance between spinous processes on lateral radiography and increased signal in the interspinous region on MR sagittal STIR sequences. Abnormal angulation, distraction, and subluxation are often recognized on initial CT study.

\subsubsection{Whiplash-Associated Disorders}

Whiplash injuries represent a separate, relatively common entity (1-4/1000), resulting from an acceleration-deceleration mechanism of injury to the neck, typically from rear-end vehicle collisions. Whiplash injury is among the leading automotive related injuries with respect to burden on patients, the healthcare system and insurance organizations [27]. The pathogenesis of whiplash complaints is still poorly understood. Injury to longitudinal ligaments, facet joints, intervertebral discs, spinal cord, and muscles has been described as possible sources of (chronic) pain. More recently, with the development of more detailed MR imaging techniques, morphologic changes of the ligaments and membranes of the craniovertebral junction, especially the alar and transverse ligaments have been described [28].

Whiplash-associated disorders (WAD) is a clinical diagnosis and describes a variety of clinical manifestations, such as neck pain immediately or $24 \mathrm{~h}$ after trauma, neck stiffness, headache, dizziness, vertigo, auditory and visual disturbances, concentration, and psychological problems. Imaging findings include osseous injuries such as bone contusions and occult fractures, ligamentous injuries (most common finding), and tears. Disc lesions and post-traumatic herniation also can occur [29]. MRI signal changes of the alar and transverse ligaments may be observed. Whether these signal abnormalities are responsible for complaints of patients having WAD remains controversial, as these signal abnormalities have also been observed in asymptomatic individuals and were not significantly associated with clinical testing and prognosis of acute whiplash injury. A recent meta-analysis could not show any association between MRI signal changes in alar and transverse ligaments and WAD [28].

\section{Key Point}

- MRI has unparalleled sensitivity to the gamut of soft tissue injuries associated with spinal trauma; however, signal changes on T2-weighted or STIR MR in the soft tissues due not equate with spinal instability.

\subsection{Injuries to the Spinal Cord}

A majority (80\%) of patients with spinal cord injury (SCI) harbor multisystem injuries [30], typically associated injuries include other bone fractures $(29.3 \%)$ and brain injury $(11.5 \%)$ [31]. Nearly all SCI damage both upper and lower motor neurons because they involve both the gray matter and descending white matter tracts at the level of injury. The American Spinal Injury Association (ASIA) has suggested a comprehensive set of standardized clinical measurements which are based upon a detailed sensory and motor examination of all dermatomes and myotomes [32]. The neurologic deficit that results from injury to the spinal cord depends primarily upon the extent of damage at the injury site and the cranial-caudal location of the damage (i.e., the neurologic level of injury or NLI); anatomically higher injuries produce a greater neurologic deficit (e.g., cervical injury = quadriparesis, thoracic injury = paraparesis). These comprehensive set of standardized clinical measurements have been adopted worldwide. Functional transection of the spinal cord is a more frequent manifestation of SCI compared to true mechanical transection which is relatively rare and confined mostly to penetrating type injuries or extensive fracture-dislocations/translocations. SCI is further categorized clinically into anterior cord syndrome, Brown-Sequard syndrome, central cord syndrome, conus medullaris syndrome, and cauda equina syndrome depending upon the site of injury and the neurologic pattern of injury. Spontaneous neurologic recovery after spinal cord injury overall is relatively poor and largely depends upon the severity of neurologic deficit identified at the time of injury. Of the different spinal cord syndromes the anterior cord syndrome has the worst prognosis of all cord syndromes, especially, if no recovery is noticed during the first $72 \mathrm{~h}$ after injury.

\subsubsection{Spinal Cord Hemorrhage}

Post-traumatic spinal cord hemorrhage or hemorrhagic contusion is defined as the presence of a discrete area of hem- 
orrhage within the spinal cord after an injury. The most common location for hemorrhage to accumulate is within the central gray matter of the spinal cord, and centered at the point of mechanical impact [33-35]. Experimental and autopsy pathologic studies have shown that the underlying lesion most often will be hemorrhagic necrosis of the spinal cord while true hematomyelia will rarely be found [36]. There are significant clinical implications if there is identification of frank hemorrhage in the cervical spinal cord following trauma on an MRI examination. Originally it was thought that detection of intramedullary hemorrhage was predictive of a complete injury. However, the increased sensitivity and spatial resolution of current MRI techniques has shown that even small amounts of hemorrhage are identifiable in incomplete lesions. Therefore, the basic construct has been altered such that the detection of a sizable focus of blood $(>4 \mathrm{~mm}$ in length on sagittal images) in the cervical spinal cord is often indicative of a complete neurological injury [37]. The anatomic location of the hemorrhage closely corresponds to the neurological level of injury and the presence of frank hemorrhage implies a poor potential for neurological recovery (Fig. 19.1) [33-35, 38-42].

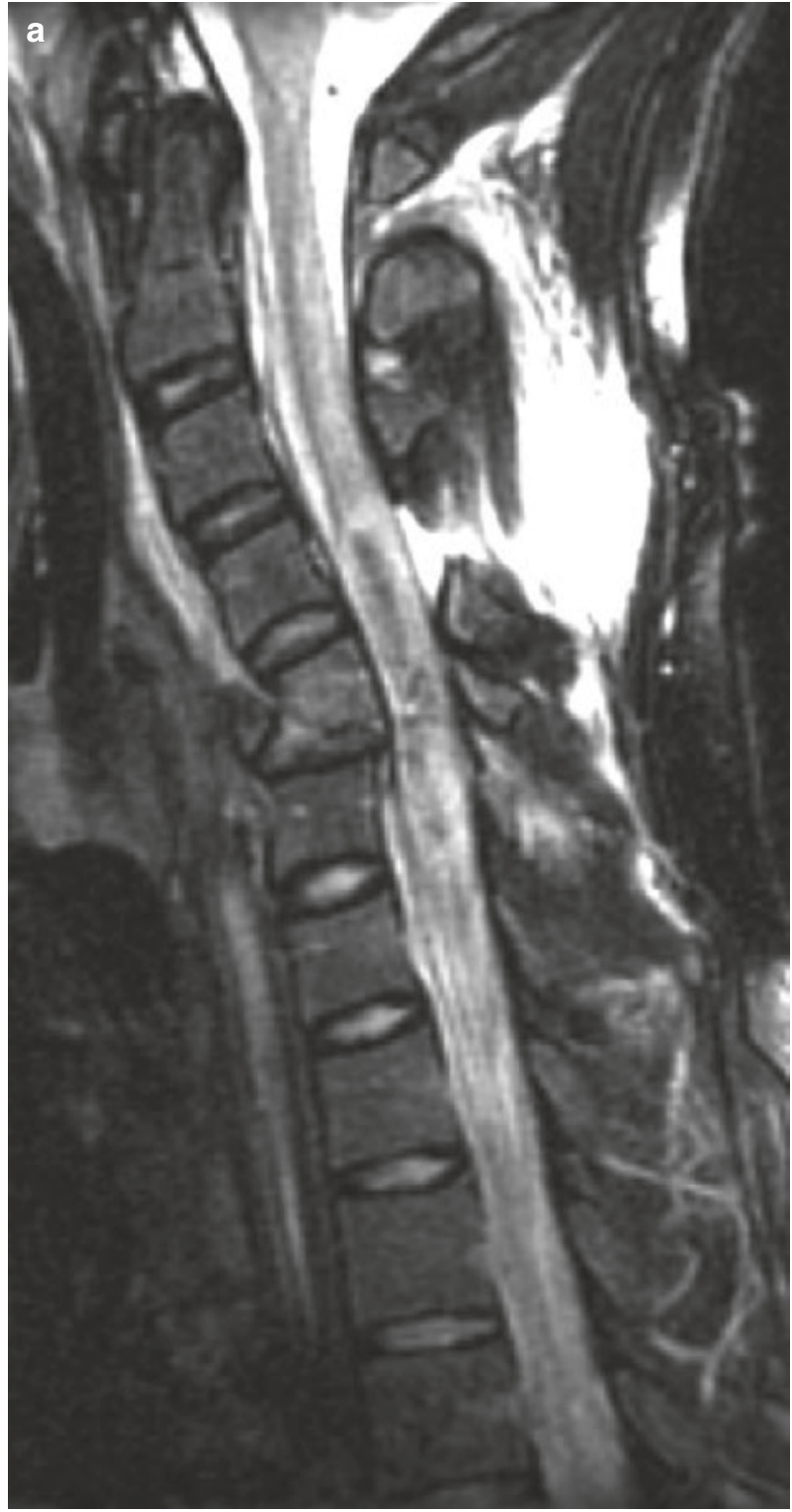

Fig. 19.1 Acute hemorrhagic spinal cord injury. (a) Sagittal STIR sequence shows that there is a flexion type injury of C5 with acute ventral angulation. The spinal cord is markedly swollen with edema spanning the entire length of the spinal cord. There is a central hemorrhagic

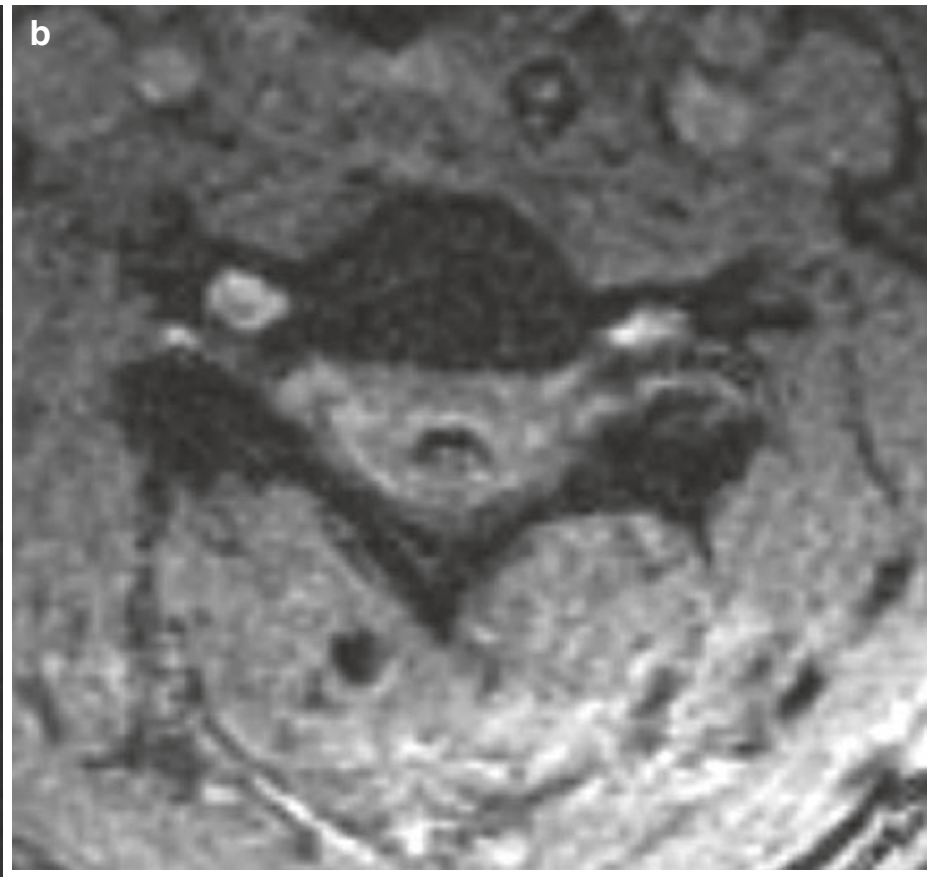

focus which is of low signal intensity that spans from C4 to C6. Note the disruption of the posterior spinal soft tissues. (b) Axial GRE image at the $\mathrm{C} 4$ level shows that the hypointense hemorrhage is confined to the central gray matter 


\subsubsection{Spinal Cord Edema}

Spinal cord edema is defined as a focus of abnormal high signal intensity seen on MRI T2-weighted images [35]. Presumably, this signal abnormality reflects a focal accumulation of intracellular and interstitial fluid in response to injury $[34,35,43,44]$. Edema is usually well defined on the mid-sagittal T2-weighted image while the axial T2-weighted images offer additional information in regard to involvement of structures in cross-section. Spinal cord edema involves a variable length of spinal cord above and below the level of injury, with discrete boundaries adjacent to uninvolved parenchyma and is invariably associated with some degree of spinal cord swelling. The length of spinal cord affected by edema is directly proportional to the degree of initial neurologic deficit [33, 42]. Notable is that spinal cord edema can occur without MRI evidence of intramedullary hemorrhage. Cord edema alone connotes a more favorable prognosis than cord hemorrhage [38].

\section{Key Point}

- The combination of intramedullary hemorrhage and/ or edema correlates with neurologic function and capacity to recover after spinal cord injury (SCI).

\subsection{Blunt Cerebrovascular Injury}

Blunt cerebrovascular injury (BCVI) to the carotid arteries (CAs) and/or vertebral arteries (VAs) is a relatively rare but potentially devastating finding in patients with a high-impact trauma to the cervical spine and/or head. Most complications of BCVI occur hours to days after the initial trauma so early identification and prompt anticoagulation is important to reduce the incidence of post-injury ischemic stroke (Fig. 19.2).

Cervical spine fractures have the strongest association with BCVI, and specific cervical spine fractures are highly predictive of BCVI. These include fractures located at the upper cervical spine $(\mathrm{C} 1-\mathrm{C} 3)$, subluxation, or involvement of the transverse foramina [45]. In the context of a carotid artery injury, it is thought that cervical hyperextension and contralateral rotation lead to stretching of the internal carotid artery (ICA) over the $\mathrm{C} 1-\mathrm{C} 3$ transverse processes, precipitating a vessel wall injury [46]. Nearly all VAIs are associated with cervical spine subluxations and fractures involving the transverse foramen [47]. Other mechanisms of BCVI include hyperflexion, a direct blow, and strangulation.

According to the Denver criteria and Western Trauma Association (WTA) guidelines, radiologic risk factors associated with BCVI include high-energy injury mechanisms with Le Fort II or III fracture patterns; basilar skull fracture with carotid canal involvement; cervical vertebral body or transverse foramen fracture, subluxation, or ligamentous injury; any fracture at $\mathrm{C} 1-\mathrm{C} 3$; closed head injuries with diffuse axonal injury and a Glasgow Coma Score of $<6$; clothesline-type injuries with associated swelling and/ or pain; or near-hanging with anoxia. Urgent screening for BCVI should be performed in these patients that may be still neurologically asymptomatic at this time [46].

With the widespread use of multi-detector CT scanners, CT angiography (CTA) has emerged as the first-line screening modality for BCVI. CTA is a cost-effective screening tool in high-risk populations, ultimately preventing the most strokes at a reasonable cost [46].

Several grading systems exist, based on imaging findings. The Denver grading scale (aka the Biffl scale) is a widely accepted and commonly used system. In grade I injury, subtle vessel wall irregularities, a dissection/intramural hematoma with $<25 \%$ luminal stenosis is observed. In grade II injury, an intraluminal thrombus or raised intimal flap is visualized, or a dissection/intramural hematoma with $>25 \%$ luminal narrowing. Grade III injury corresponds to traumatic pseudoaneurysms, observed as a variable-sized outpouching of the vessel wall. Grade IV injury represents a complete vessel occlusion, usually tapering in the CA and quite abrupt in the VA. Vessel transection represents a grade $\mathrm{V}$ injury, observed as free contrast extravasation into the surrounding tissues, or into the adjacent vein in the form of an arteriovenous fistula (AVF) [46, 47].

\section{Key Point}

- Occult vertebral artery injury (dissection or occlusion) can occur in association with spinal trauma even without the presence of spinal cord injury or fracture.

\subsection{Injuries to the Pediatric Spine and Spinal Cord}

Spinal injuries are generally less common in the pediatric population compared to adults with cervical spine injuries being most frequent spine injury of all spine injuries occurring in up to $40-60 \%$ of all injuries in children. The etiology varies depending on the age of the child. The most common cause of pediatric cervical spine injury is a motor vehicle accident, but also obstetric complication, fall, and child abuse are known causes. In the adolescent, sports and diving accidents are other well-known causes. The specific biomechanics of the pediatric cervical spine leads to a different distribution of injuries and distinct radiological features and represent a distinct clinical entity compared to those seen in adults. Young children have a propensity for injuries to the CCJ, upper cervical injuries (i.e., cranial base to $\mathrm{C} 2$ ) whereas older children are prone to lower cervical injuries 


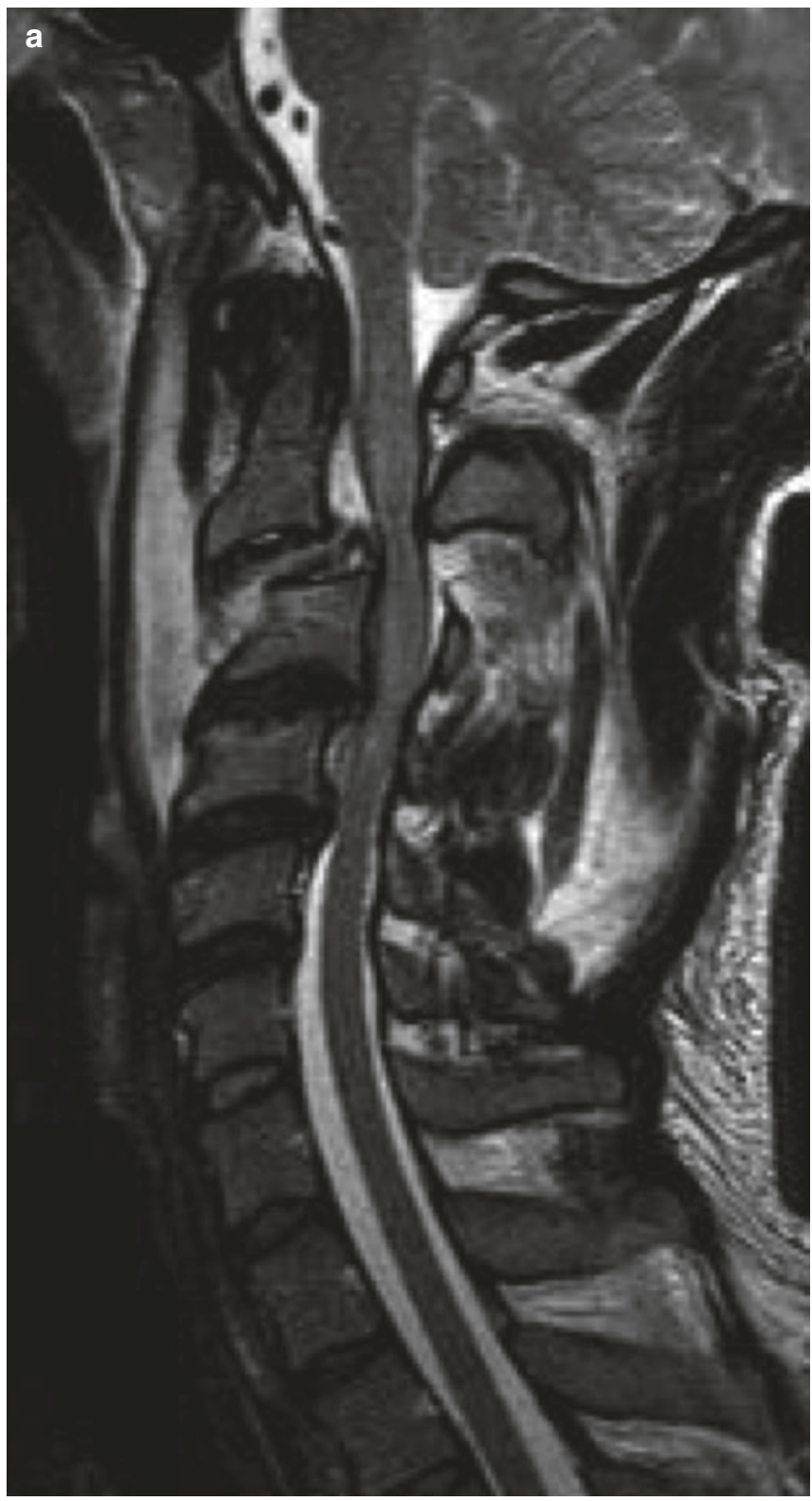

Fig. 19.2 Traumatic thrombosis/dissection of the bilateral vertebral arteries. (a) Sagittal T2-weighted image in a 32 y/o male after a skateboarding accident. Traumatic subluxation of $\mathrm{C} 2-3$ with circumferential disruption of the disco-ligamentous complex and spinal cord compres-

similar to those seen in adults. The spinal cervical injuries in children $<8$ years of age demonstrate a high incidence of subluxation without fractures. The biomechanical differences are explained by the relative ratio of the size of the cranium to the body in the young child, lack of ligamentous stability, poor muscle strength, and increased forces relative to the older child and adult. Children are also more prone to spinal cord injury with otherwise normal radiographs so called SCIWORA (spinal cord injury without radiographic abnormality) compared to adults. This is especially evident in children younger than 9 years of age where there is a high incidence of reported complete cord injuries associated

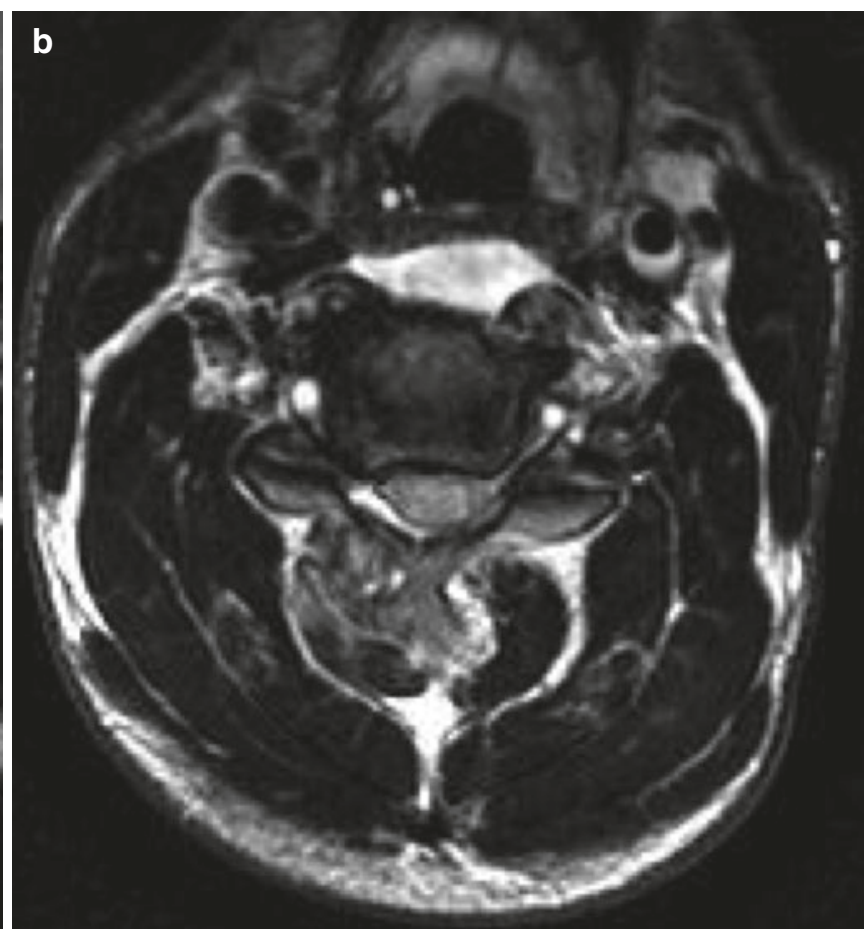

sion/edema. (b) Axial T2-weighted image at the $\mathrm{C} 2-3$ level show high signal in both foramen transversarium from clot/slow flow in both vertebral arteries

with SCIWORA. Suggested mechanisms of the SCIWORA include hyperextension or flexion injuries to the immature and the inherently elastic spine, which is vulnerable to external forces and allows for significant intersegmental movement and transient soft disc protrusion, resulting in distraction injuries, and/or ischemic injury of the spinal cord [48]. The elasticity of the spine allows it to stretch up to $5 \mathrm{~cm}$ before rupture, whereas the spinal cord, which is anchored to the brachial plexus superiorly and the cauda equina inferiorly, ruptures after 4-6 mm of traction [49]. As MRI is readily capable of detecting the soft tissue injury component, the concept of SCIWORA is less relevant. 
The imaging algorithm for pediatric spinal trauma is somewhat different than that for adults. MDCT is used more judiciously due to radiation exposure considerations and at many places lower dose radiography is often utilized initially. MRI is always used if there is a consideration of a pure soft tissue injury or neurologic deficit.

\section{Key Point}

- Patterns of injury in the pediatric population are tremendously different than adults; the imaging algorithm is unique.

\subsection{Neurologic Recovery after Spinal Cord Injury}

Although there are no pharmacologic "cures" for spinal cord injury, spontaneous neurologic recovery after injury can occur and it largely depends upon the severity of the initial neurologic deficit, the neurologic level of injury, patient age, and co-morbidities. Very few patients with a neurologically complete (i.e., no motor or sensory function below the injury level) actually regain any useful function below the injury level although most patients will spontaneously improve by one neurologic level (e.g., a C5 level spontaneously descends to a C6 level). Even these small improvements can have a substantial impact on a patients' capacity to function independently.

The role of MRI to predict capacity for spontaneous neurologic recovery after cervical SCI has been evaluated. Although there is considerable overlap in results some general characterizations about the MRI appearance of SCI and neurologic recovery are evident. Intramedullary hemorrhage four millimeters or greater is equated with a severe neurologic deficit and a poor prognosis. Cord edema alone is indicative of a mild to moderate initial neurological deficit and a better capacity for spontaneous neurological improvement. The length of the cord lesion may also correlate with the initial deficit and in the neurological outcome. Newer MRI techniques such as diffusion tensor imaging (DTI) (Fig. 19.3) have shown great promise not only in stratification of neurologic injury but has also been shown to have benefits in predicting recovery [50-52]. As novel pharmacologic therapies for SCI are developed and tested, MRI will likely play a more essential role in characterizing the injury and helping to select patients for clinical trials.

\section{Key Point}

- MRI findings of SCI have shown promise as a surrogate for the neurologic examination; better prognostic information is attained when MR imaging patterns are used in combination with the clinical examination.
Fig. 19.3 Disruption of the lateral cortical spinal tracts on tractography. Diffusion tractography performed on a lesioned rat spinal cord at 9.4T shows disruption of the fiber tracts in the lateral column following a lesion in the lateral funiculus. (Image courtesy of Eric D. Schwartz MD)

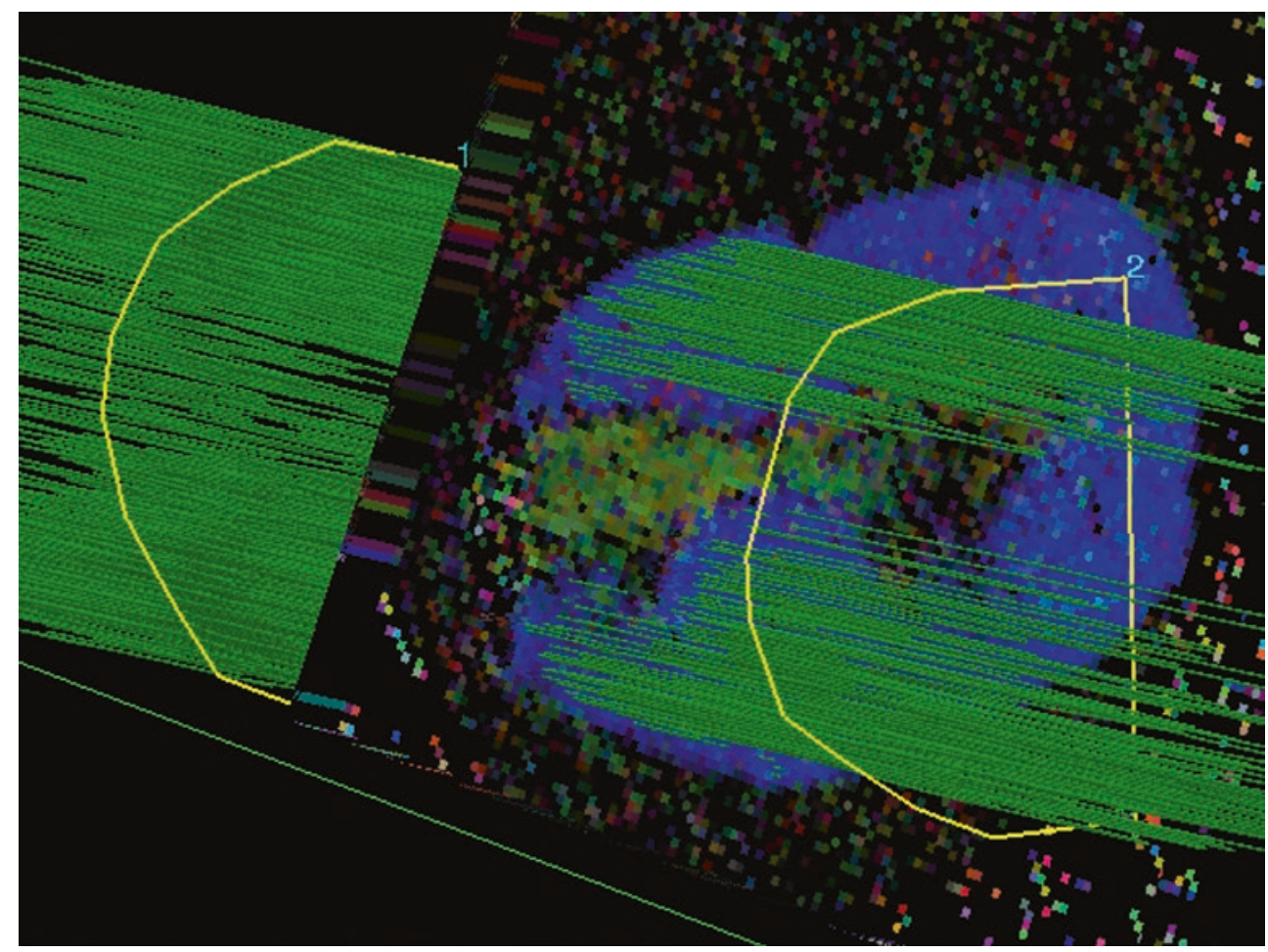




\subsection{Concluding Remarks}

The demographic of adult spinal trauma and spinal cord injury has changed over the last several decades noting a considerable increase in prevalence in the elderly. Safety measures in sports and motor vehicles have drastically reduced the incidence of spinal cord injury in the young adult and middle age population. Imaging algorithms for spinal trauma have also changed in the past few decades with greater reliance on MDCT over radiography in adults and greater emphasis towards minimizing radiation dose in the injured pediatric population. While there have been tremendous improvements in orthopedic stabilization methods through novel instrumentation/fusion techniques, there are still no known cures for paralysis. While spinal cord injury was previously considered to be a fatal disease with patient succumbing to injury at the scene or from long term complications, most SCI patients now survive and many return to meaningful lives while living with their disability. This is principally attributed to revolutionary improvements in rehabilitation and chronic care for the SCI patient.

\section{Take Home Messages}

- Spinal trauma and spinal cord injury are prevalent worldwide.

- Spinal trauma is now more common in the elderly due to falls.

- MDCT has supplemented radiography.

- MRI reveals the soft tissue components of injury including damage to the spinal cord.

- MR signal changes with whiplash-associated disorders are inconsistently present.

\section{References}

1. Riascos R, Bonfante E, Cotes C, Guirguis M, Hakimelahi R, West C. Imaging of atlanto-occipital and atlantoaxial traumatic injuries: what the radiologist needs to know. Radiographics. 2015;35(7):2121-34. https://doi.org/10.1148/rg.2015150035.

2. Hill MW, Dean SA. Head injury and facial injury: is there an increased risk of cervical spine injury? J Trauma. 1993;34: 549-54.

3. Pope AM, Tarlov AR. Disability in America: toward a national agenda for prevention. Washington: National Academy Press; 1991.

4. Riggins RS, Kraus JF. The risk of neurological damage with fractures of the vertebrae. J Trauma. 1997;17:126-30.

5. Castellano V, Bocconi FL. Injuries of the cervical spine with spinal cord involvement (myelic fractures): statistical considerations. Bull Hosp J Dis Orthop Inst. 1970;31:188-98.

6. Rogers WA. Fractures and dislocations of the cervical spine; an endresult study. J Bone Joint Surg. 1957;39:341-51.
7. Diaz JJ Jr, Gillman C, Morris JA Jr, et al. Are five-view plain films of the cervical spine unreliable? A prospective evaluation in blunt trauma in patients with altered mental status. J Trauma. 2003;55:658-63.

8. Griffen MM, Frykberg ER, Kerwin AJ, et al. Radiographic clearance of blunt cervical spine injury: plain radiograph or computed tomography scan? J Trauma. 2003;55:222-6.

9. Holmes JF, Mirvis SE, Panacek EA, et al. Variability in computed tomography and magnetic resonance imaging in patients with cervical spine injuries. J Trauma. 2002;53:524-9.

10. Kligman M, Vasili C, Roffman M. The role of computed tomography in cervical spine injury due to diving. Arch Orthop Trauma Surg. 2001;121:139-41.

11. Schenarts PJ, Diaz J, Kaiser C, et al. Prospective comparison of admission computed tomographic scan and plain films of the upper cervical spine in trauma patients with altered mental status. J Trauma. 2001;51:663-8.

12. Berne JD, Velmahos GC, El Tawil Q, et al. Value of complete cervical helical computed tomographic scanning in identifying cervical spine injury in the unevaluable blunt trauma patient with multiple injuries: a prospective study. J Trauma. 1999;47:896-902.

13. Van Goethem JW, Maes M, Özsarlak Ö, et al. Imaging in spinal trauma. Eur Radiol. 2005;15(3):582-90.

14. Flanders AE, Schaefer DM, Doan HT, et al. Acute cervical spine trauma; correlation of MR imaging findings with degree of neurological deficit. Radiology. 1990;177:25-33.

15. Sliker CW, Mirvis SE, Shanmuganathan K. Assessing cervical spine stability in obtunded blunt trauma patients; review of medical literature. Radiology. 2005;234:733-9.

16. Wilmink JT. MR imaging of the spine: trauma and degenerative disease. Eur Radiol. 1999;9:1259-66.

17. Hogan GJ, Mirvis SE, Shanmuganathan K, Scalea TM. Exclusion of unstable cervical spine injury in obtunded patients with blunt trauma: is MR imaging needed when multi-detector row CT findings are normal? Radiology. 2005;237:106-13.

18. Denis F. The three column spine and its significance in the classification of acute thoracolumbar spinal injuries. Spine. 1983;8(8):817-31.

19. Mcafee PC, Yuan HA, Fredrickson BE, et al. The value of computed tomography in thoracolumbar fractures. An analysis of one hundred consecutive cases and a new classification. J Bone Joint Surg Am. 1983;65(4):461-73.

20. Magerl F, Aebi M, Gertzbein SD, et al. A comprehensive classification of thoracic and lumbar injuries. Eur Spine J. 1994;3(4): 184-201.

21. Lee JY, Vaccaro AR, Lim MR, et al. Thoracolumbar injury classification and severity score: a new paradigm for the treatment of thoracolumbar spine trauma. J Orthop Sci. 2005;10(6):671-5.

22. Dvorak MF, Fischer CG, Fehlings MG, Rampersaud YR, Öner FC, Aarabi B, Vaccaro AR. The surgical approach to subaxial cervical spine injuries: an evidence-based algorithm based on the classification system. Spine. 2007:32(23):2620-9.

23. Kepler CK, Vaccaro AR, Koerner JD, Dvorak MF, Kandziora F, Rajasekaran S, Aarabi B, Vialle LR, Fehlings MG, Schroeder GD, Reinhold M, Schnake KJ, Bellabarba C, Cumhur Öner F. Reliability analysis of the AOSpine thoracolumbar spine injury classification system by a worldwide group of naïve spinal surgeons. Eur Spine J. 2016;25(4):1082-6.

24. Rogers LF. The roentgenographic appearances of transverse or chance fractures of the spine: the seat belt fracture. Am J Roentgenol. 1971;111:844-9.

25. Gertzbein SD. Scoliosis Research Society: multicenter spine fracture study. Spine. 1992;17:528-40.

26. Diaz JJ, Aulino JM, Collier B, et al. The early work-up for isolated ligamentous injury of the cervical spine; does computed tomography scan have a role. J Trauma. 2005;59:897-904. 
27. Sarrami P, Armstrong E, Naylor JM, Harris IA. Factors predicting outcome in whiplash injury: a systematic meta-review of prognostic factors. J Orthop Traumatol. 2017;18:9-16.

28. Li Q, Shen H, Li M. Magnetic resonance imaging signal changes of alar and transverse ligaments not correlated with whiplashassociated disorders. A meta-analysis of case-control studies. Eur Spine J. 2013;22:14-20.

29. Boban J, Thurnher MM, Van Goethem JW. Spine and spinal cord trauma. In: Barkhof $\mathrm{F}$, et al., editors. Clinical neuroradiology. Cham: Springer; 2018.

30. Burney RE, Maio RF, Maynard F, et al. Incidence, characteristics, and outcome of spinal cord injury at trauma centers in North America. Arch Surg. 1993;128:596-9.

31. Dawodu ST. Spinal cord injury - definition, epidemiology, pathophysiology. Emedicine; 2009.

32. American Spinal Injury Association. International standards for neurological classifications of spinal cord injury. Revised ed. Chicago: American Spinal Injury Association; 2000. p. 1-23.

33. Bondurant FJ, Cotler HB, Kulkarni MV, et al. Acute spinal cord injury. A study using physical examination and magnetic resonance imaging. Spine. 1990;15(3):161-8.

34. Flanders AE, Spettell CM, Friedman DP, Marino RJ, Herbison GJ. The relationship between the functional abilities of patients with cervical spinal cord injury and the severity of damage revealed by MR imaging. AJNR Am J Neuroradiol. 1999;20(5):926-34.

35. Kulkarni MV, McArdle CB, Kpanicky D, et al. Acute spinal cord injury: MR imaging at 1.5 T. Radiology. 1987;164(3):837-43.

36. Schouman-Claeys E, Frija G, Cuenod CA, et al. MR imaging of acute spinal cord injury: results of an experimental study in dogs. AJNR Am J Neuroradiol. 1990;11(5):959-65.

37. Boldin C, Raith J, Fankhauser F, Haunschmid C, Schwantzer G, Schweighofer F. Predicting neurologic recovery in cervical spinal cord injury with postoperative MR imaging. Spine (Phila Pa 1976). 2006;31(5):554-9.

38. Flanders AE, Spettell CM, Tartaglino LM, Friedman DP, Herbison GJ. Forecasting motor recovery after cervical spinal cord injury: value of MR imaging. Radiology. 1996;201(3):649-55.

39. Cotler HB, Kulkarni MV, Bondurant FJ. Magnetic resonance imaging of acute spinal cord trauma: preliminary report. J Orthop Trauma. 1988;2(1):1-4.

40. Sato T, Kokubun S, Rijal KP, et al. Prognosis of cervical spinal cord injury in correlation with magnetic resonance imaging. Paraplegia. 1994;32(2):81-5.
41. Marciello MA, Flanders AE, Herbison GJ, et al. Magnetic resonance imaging related to neurologic outcome in cervical spinal cord injury. Arch Phys Med Rehabil. 1993;74(9):940-6.

42. Talbott JF, Whetstone WD, Readdy WJ, Ferguson AR, Bresnahan JC, Saigal R, Hawryluk GW, Beattie MS, Mabray MC, Pan JZ, Manley GT, Dhall SS. The brain and spinal injury center score: a novel, simple, and reproducible method for assessing the severity of acute cervical spinal cord injury with axial T2-weighted MRI findings. J Neurosurg Spine. 2015;23(4):495-504.

43. Goldberg AL, Rothfus WE, Deeb ZL, et al. The impact of magnetic resonance on the diagnostic evaluation of acute cervicothoracic spinal trauma. Skelet Radiol. 1988;17(2):89-95.

44. Wittenberg RH, Boetel U, Beyer HK. Magnetic resonance imaging and computer tomography of acute spinal cord trauma. Clin Orthop Relat Res. 1990;260:176-85.

45. Kopelman TR, Leeds S, Berardoni NE, et al. Incidence of blunt cerebrovascular injury in low-risk cervical spine fractures. Am J Surg. 2011;202:684-8; discussion 688-9.

46. Rutman AM, Vranic JE, Mossa-Basha M. Imaging and management of blunt cerebrovascular injury. Radiographics. 2018;38:542-63.

47. Cothren CC, Moore EE, Biffle WL, et al. Cervical spine fracture patterns predictive of blunt vertebral artery injury. J Trauma. 2003;55:811-3.

48. Kriss VM, Kriss TC. SCIWORA (spinal cord injury without radiographic abnormality) in infants and children. Clin Pediatr (Phila). 1996;35:119-24.

49. Manary MJ, Jaffe DM. Cervical spine injuries in children. Pediatr Ann. 1996;25:423-8.

50. Poplawski MM, Alizadeh M, Oleson CV, Fisher J, Marino RJ, Gorniak RJ, Leiby BE, Flanders AE. Application of diffusion tensor imaging in forecasting neurological injury and recovery after human cervical spinal cord injury. J Neurotrauma. 2019;36:3051. https://doi.org/10.1089/neu.2018.6092.

51. Shanmuganathan K, Zhuo J, Chen HH, Aarabi B, Adams J, Miller C, Menakar J, Gullapalli RP, Mirvis SE. Diffusion tensor imaging parameter obtained during acute blunt cervical spinal cord injury in predicting long-term outcome. J Neurotrauma. 2017;34:2964-71.

52. Schaefer DM, Flanders A, Northrup BE, et al. Magnetic resonance imaging of acute cervical spine trauma. Correlation with severity of neurologic injury. Spine. 1989;14(10):1090-5.

Open Access This chapter is licensed under the terms of the Creative Commons Attribution 4.0 International License (http://creativecommons. org/licenses/by/4.0/), which permits use, sharing, adaptation, distribution and reproduction in any medium or format, as long as you give appropriate credit to the original author(s) and the source, provide a link to the Creative Commons license and indicate if changes were made.

The images or other third party material in this chapter are included in the chapter's Creative Commons license, unless indicated otherwise in a credit line to the material. If material is not included in the chapter's Creative Commons license and your intended use is not permitted by statutory regulation or exceeds the permitted use, you will need to obtain permission directly from the copyright holder. 